\title{
Heavy-ion Physics (ATLAS)
}

\author{
Mariusz Przybycien ${ }^{1, a}$ (on behalf of the ATLAS Collaboration) \\ ${ }^{1}$ AGH University of Science and Technology, Faculty of Physics and Applied Computer Science \\ Al. Mickiewicza 30, 30-059 Cracow, Poland
}

\begin{abstract}
The ATLAS experiment at the Large Hadron Collider has undertaken a broad physics program to probe and characterize the hot nuclear matter created in relativistic heavy-ion collisions. This talk presents recent results on production of electroweak bosons and quarkonium, charged particles and jets, bulk particle collectivity and electromagnetic processes in ultra-peripheral collisions, from $\mathrm{Pb}+\mathrm{Pb}$ and $p+\mathrm{Pb}$ systems.
\end{abstract}

\section{Introduction}

The Quark-Gluon Plasma (QGP) was first observed in experiments at RHIC [1-4] and since then it has been intensively studied in relativistic heavy-ion (HI) collisions. Significantly higher collision energies available at the LHC compared to RHIC, result in increased volume, lifetime and temperature of QGP. We are particularly interested in understanding of the collision geometry and centrality as well as in how the free nucleon parton distribution functions are modified in the nuclear environment. One can study properties of QGP using hard probes of different scales (electroweak bosons, jets, heavy quarks) or measuring parameters describing collective behaviour of the medium. Hard probes are produced early in the $\mathrm{HI}$ collision, in a process whose cross section is not changed by the presence of strongly interacting medium, i.e. it can be calculated using perturbative QCD. Passing through the medium hard probes interact weakly or strongly with it providing information on its properties. Due to their large electric charge heavy-ions are also strong sources of high energy photons. Thus, in ultra-peripheral collisions, in which strong interactions are suppressed, one can study two-photon and photo-nuclear interactions. A review of HI results from LHC experiments based on Run 1 data can be found in Ref. [5]. In these proceedings an overview of selected recent HI results from the ATLAS experiment [6] is presented mainly based on $\mathrm{Pb}+\mathrm{Pb}$ and $p+\mathrm{Pb}$ collision data at $\sqrt{s_{\mathrm{NN}}}=5.02 \mathrm{TeV}$.

Yields of hard processes in HI collisions are expected to scale with the number of binary nucleonnucleon collisions, $N_{\text {coll }}$, which depends on the centrality of the collision. In the central collisions, when the overlap of nuclei is large, one expects a high number of $N_{\text {coll }}$ and a high number of nucleons participating in the collision, $N_{\text {part }}$. On the other hand, in more peripheral collisions, when nuclei overlap only partly, both $N_{\text {coll }}$ and $N_{\text {part }}$ are expected to be smaller.

A proxy to the QGP volume produced in a collision is the collision centrality. In ATLAS centrality of events is determined by the sum of transverse energy deposited in the forward calorimeter (FCal) covering the pseudorapidity range $3.2<|\eta|<4$.8. In case of $\mathrm{Pb}+\mathrm{Pb}$ collisions the sum on both sides and in case of $p+\mathrm{Pb}$ collisions the sum on the $\mathrm{Pb}$-going side only are taken into account $[7,8]$. The

a e-mail: Mariusz.Przybycien@agh.edu.pl 
centrality intervals are expressed in percentiles of the total inelastic non-Coulomb $\mathrm{Pb}+\mathrm{Pb}$ or $p+\mathrm{Pb}$ cross sections, respectively. For the correlation between a centrality interval and the average number of participants, $\left\langle N_{\text {part }}\right\rangle$, the Glauber model is used for $\mathrm{Pb}+\mathrm{Pb}$ collisions and both the Glauber and Glauber-Gribov colour fluctuation [9] models for $p+\mathrm{Pb}$ collisions.

A variable often used to quantify the modification of rates of hard processes in a nuclear environment with respect to $p p$ collisions is the nuclear modification factor:

$$
R_{\mathrm{AA}}=\frac{1}{\left\langle T_{\mathrm{AA}}\right\rangle} \frac{1 / N_{\mathrm{evt}} \mathrm{d}^{2} N_{\mathrm{A}+\mathrm{A}} / \mathrm{d} \eta \mathrm{d} p_{\mathrm{T}}}{\mathrm{d}^{2} \sigma_{p p} / \mathrm{d} \eta \mathrm{d} p_{\mathrm{T}}}
$$

where in the numerator is the yield of a given process per minimum-bias $(\mathrm{MB})$ event in nucleusnucleus $(\mathrm{A}+\mathrm{A})$ collisions, possibly in a given centrality interval, and in the denominator the $p p$ cross section for the same process. The $T_{\mathrm{AA}}$ is the mean nuclear thickness function and is a measure of the nuclear overlap, i.e. of the number of nucleons which can participate in the hard scattering processes.

\section{Electroweak bosons and charmonia}

Electroweak (EW) bosons being colourless do not interact strongly with QGP, and their production rates are therefore expected to be directly sensitive to the overall thickness of the colliding nuclear matter. The rates are also expected to be sensitive to modifications of the partonic structure of nucleons bound in a nucleus. These effects include nuclear shadowing (depletion of the parton densities at low Bjorken $x$ ), antishadowing (an enhancement at moderate $x$ ), the EMC effect and Fermi motion [10].

ATLAS has studied production of $W$ and $Z$ bosons in both $\mathrm{Pb}+\mathrm{Pb}$ collisions at $\sqrt{s_{\mathrm{NN}}}=2.76 \mathrm{TeV}$ $[11,12]$ and in $p+\mathrm{Pb}$ collisions at $\sqrt{s_{\mathrm{NN}}}=5.02 \mathrm{TeV}[13,14]$. Recently also production of $Z$ bosons in the di-muon decay channel in $\mathrm{Pb}+\mathrm{Pb}$ collisions at $\sqrt{s_{\mathrm{NN}}} 5.02 \mathrm{TeV}$ has been measured [15]. The yields per event of $Z$ bosons as a function of rapidity measured in different centrality intervals and compared to the $p p$ baseline measurement are shown in Fig. 1(left). The yields scaled by the average $T_{\mathrm{AA}}$ per centrality interval are in good agreement with the model, with the exception of the most peripheral class of events which shows a slight excess compared to the model prediction and the $p p$ measured data points. The rapidity differential nuclear modification factor $R_{\mathrm{AA}}$, as shown in the lower panel, is
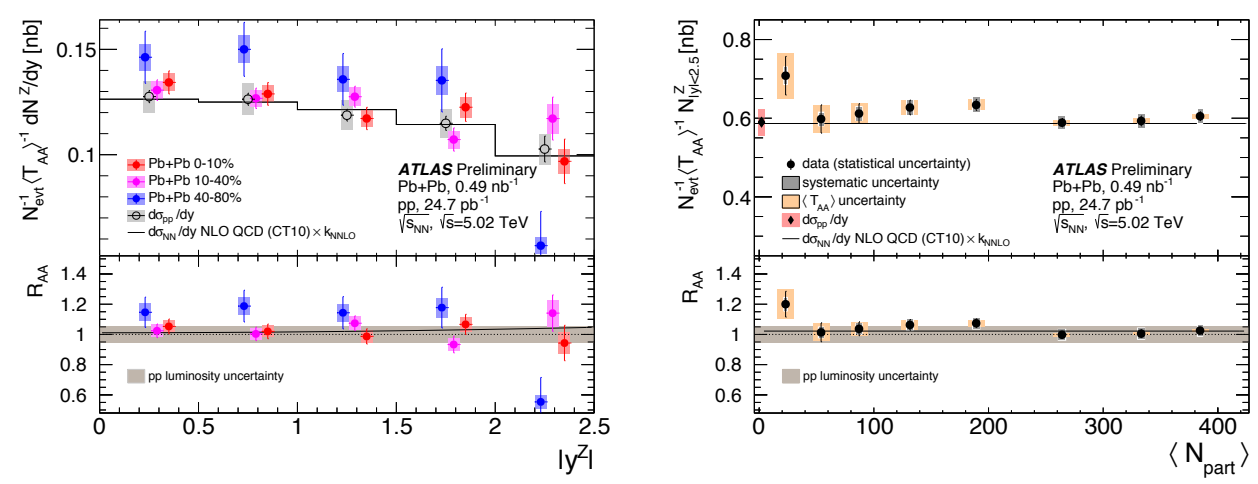

Figure 1. (left) $Z$-boson yield per event in three centrality intervals divided by $T_{\mathrm{AA}}$ (filled circles) in $\mathrm{Pb}+\mathrm{Pb}$ colisions at $5.02 \mathrm{TeV}$ and differential cross section measurement in $p p$ (open circles) as a function of $\left|y^{Z}\right|$. Lower panel: $R_{\mathrm{AA}}$ in the three centrality intervals as a function of $\left|y^{Z}\right|$. (right) $Z$-boson yield per event in three centrality intervals divided by $T_{\mathrm{AA}}$ (circles) as a function of $\left\langle N_{\text {part }}\right\rangle$ and differential cross section measurement in $p p$ (diamonds) plotted at $N_{\text {part }}=2$. Lower panel: $R_{\mathrm{AA}}$ as a function of $\left\langle N_{\text {part }}\right\rangle$, [15]. 
close to unity for all centrality classes within the measured uncertainties. The peripheral $40-80 \%$ bin is higher than the more central bins; when integrated over measured rapidity, it is different from unity by approximately 1.5 standard deviations of statistical and systematic uncertainties. The yield per event of $Z$ bosons as a function of $N_{\text {part }}$ inside $\left|y^{Z}\right|<2.5$ in the $\mathrm{Pb}+\mathrm{Pb}$ collision is shown in the top panel of Fig. 1(right). The measured integrated cross section in $p p$ collisions at the same energy is also shown. The NLO QCD (CT10) $\times k_{\text {NNLO }}$ prediction is shown along with the data and demonstrates good agreement with the measurement. The lower panel shows the $N_{\text {part }}$ dependence of the $R_{\mathrm{AA}}$. The valuee of the $R_{\mathrm{AA}}$ is consistent with unity within the statistical and total systematic uncertainty. The most peripheral point is higher than any other point but also has the largest statistical and $\left\langle T_{\mathrm{AA}}\right\rangle$ uncertainties. It is approximately 1.5 standard deviation of the combined uncertainty away from unity.

It is well known that charmonia, bound states of $c$ and $\bar{c}$ quarks, could be a unique probe to study QGP created in HI collisions [16]. Suppression of $c \bar{c}$ bound states is expected due to Debye screening of the quark colour charge in a hot plasma when the Debye radius becomes smaller than the quarkonia binding radius. On the other hand an enhancement is also expected at low transverse momentum due to recombination of produced charm quarks and anti-quarks from the medium.

The ATLAS experiment has measured production on $J / \Psi$ and $\Psi(2 S)$ mesons in $\mathrm{Pb}+\mathrm{Pb}$ collisions at $\sqrt{s_{\mathrm{NN}}}=5.02 \mathrm{TeV}$ [17]. In the following, charmonium states produced directly in the hard interaction before QGP is produced are refered to as 'prompt' and charmonium states produced in the decay chain of $b$-hadrons are refered to as 'non-prompt'. While formation of $c \bar{c}$ bound states is inhibited by colour screening, the suppression of $b$-quark production is attributed to energy loss of propagating $b$-quarks by collisional and radiative processes. So, there is no a priori reason to expect a modification of prompt charmonia production that is similar to the modification of non-prompt production. In Fig. 2(left) the nuclear modification factor is presented as a function of $p_{\mathrm{T}}$ for production of prompt and non-prompt $J / \Psi$ mesons for $|y|<2$ and for the centrality interval from 0 to $80 \%$. It can be seen that the production of $J / \Psi$ mesons is strongly suppressed in $\mathrm{Pb}+\mathrm{Pb}$ collisions. In the kinematic range plotted, as a function of $p_{\mathrm{T}}$, the nuclear modification factor for both prompt and non-prompt meson production is seen to be in the range $0.2<R_{\mathrm{AA}}<0.6$. A small increase in $R_{\mathrm{AA}}$ with increasing $p_{\mathrm{T}}$ is observed for the prompt $J / \Psi$ production, while the non-prompt $J / \Psi$ are seen to be constant in $p_{\mathrm{T}}$ within the uncertainties. In Fig. 2(right) the nuclear modification factor is presented as a function of centrality expressed as the number of participants $N_{\text {part }}$ for production of prompt and non-prompt $J / \Psi$ mesons for rapidity $|y|<2$ and for $9<p_{\mathrm{T}}<40 \mathrm{GeV}$. It can be seen that the production of $J / \Psi$ mesons is most strongly suppressed in central collisions, as expected. In the kinematic range plotted, as a function of centrality, the nuclear modification factor for both prompt and non-prompt meson production is seen to be in the range $0.2<R_{\mathrm{AA}}<1$, with the smallest value at $R_{\mathrm{AA}} \approx 0.2$ for
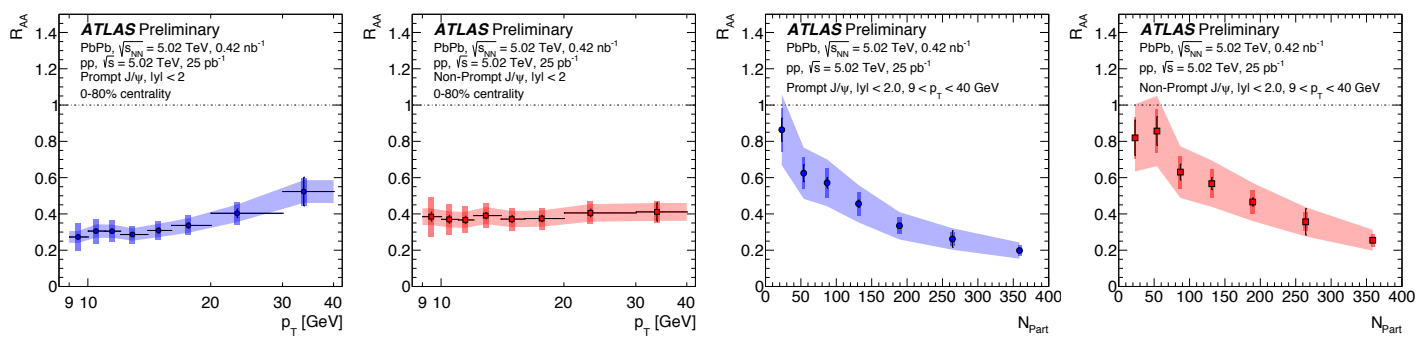

Figure 2. The nuclear modification factor $R_{\mathrm{AA}}$ as a function of (left) transverse momentum $p_{\mathrm{T}}$ for $|y|<2$ and with centrality $0-80 \%$, and (right) centrality expressed in terms of $N_{\text {part }}$ for $9<p_{\mathrm{T}}<40 \mathrm{GeV}$ and $|y|<2$, for the prompt and non-promt $J / \Psi$ mesons, [17]. 
the most central collisions for both prompt and non-prompt $J / \Psi$ mesons. Suppression by a factor of around five for both prompt and non-prompt $J / \Psi$ mesons in central collisions is a striking signature of a strong influence of the hot dense medium on the particle production processes. While the production of prompt $J / \Psi$ mesons is found to be suppressed slightly more in the mid-centrality region, the two classes of meson production have essentially the same pattern. This may not be expected, because the two classes are believed to have quite different physical origins: the non-prompt production should be dominated by $b$-quark processes that extend far outside the deconfined medium, whereas the prompt production happens predominantly deep within the medium. The measured ratio $R_{\mathrm{AA}}^{\Psi(2 S)} / R_{\mathrm{AA}}^{J / \Psi}$ (not shown) is consistent with unity for the non-prompt mesons, while the values observed for the prompt mesons are below unity. This result is consistent with what would be expected for $b$-quarks in the hot dense medium with the same behaviour for both mesons, while composite mesons formed in the hot dense medium are affected differently. In particular, the $\Psi(2 S)$ meson is suppressed more than the $J / \Psi$ meson, a pattern consistent with the lower binding energy of the $\Psi(2 S)$ meson causing it to have a lower formation and survival probability in the hot dense medium for the values of $p_{\mathrm{T}}^{\Psi(n S)}$ sampled in this measurement.

\section{Charged-particle spectra and jets}

One of the predicted consequences of QGP formation is 'jet quenching' that refers to the modification of parton showers initiated by hard-scattering processes which take place in QGP. Charged particle spectra, jet yields and jet fragmentation are three of the most important, and complementary, observables used to understand the mechanism of jet energy loss.

ATLAS has measured charged hadron spectra in $\mathrm{Pb}+\mathrm{Pb}$ collisions at $\sqrt{s_{\mathrm{NN}}}=5.02 \mathrm{TeV}$ over the full $|\eta|<2.5$ range as a function of transverse momentum up to $p_{\mathrm{T}}=300 \mathrm{GeV}$ [18]. Figure 3(left) shows the nuclear modification factor $R_{\mathrm{AA}}$ as a function of charged-particle $p_{\mathrm{T}}$ in five centrality intervals: $0-5 \%, 10-20 \%, 30-40 \%, 50-60 \%$ and $60-80 \%$. The measured $R_{\mathrm{AA}}$ shows a characteristic non-flat $p_{\mathrm{T}}$ shape which becomes more pronounced for more central collisions. It first increases with increasing $p_{\mathrm{T}}$ reaching a maximum at $p_{\mathrm{T}} \approx 2 \mathrm{GeV}$, a feature commonly associated with the Cronin effect [19], i.e. hardening of $p_{\mathrm{T}}$ spectrum in HI collisions relative to $p p$ collisions, often understood to be due to multiple scattering of partons. At higher $p_{\mathrm{T}}, R_{\mathrm{AA}}$ values decrease reaching a minimum at $p_{\mathrm{T}} \approx 7 \mathrm{GeV}$, where the charged-particle suppression is strongest. The rate of charged particles
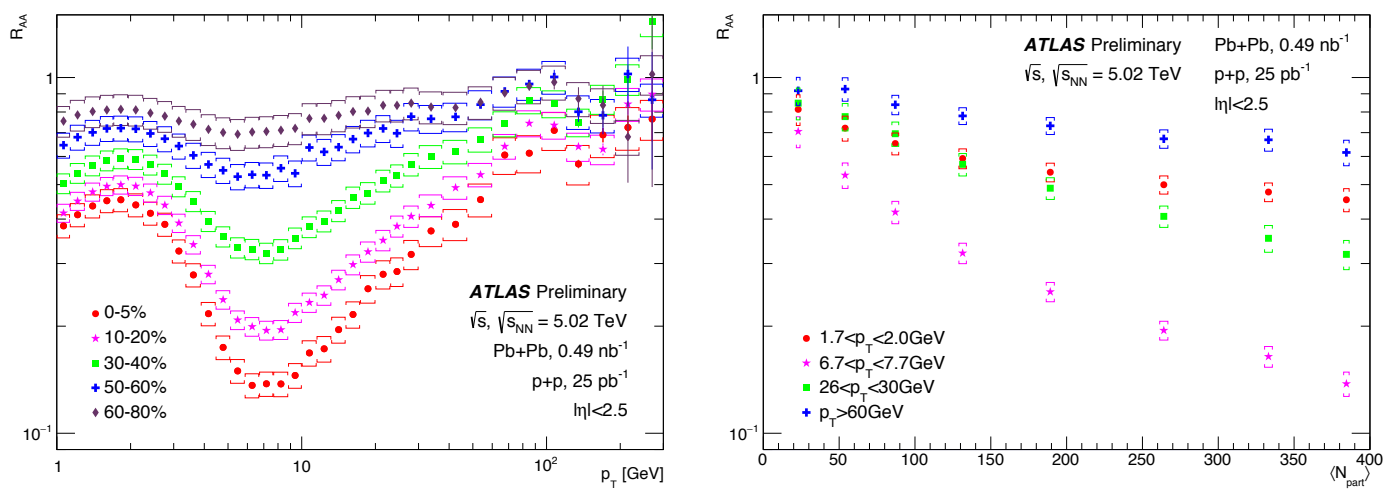

Figure 3. Nuclear modification factor $R_{\mathrm{AA}}$ for charged hadrons measured in $\mathrm{Pb}+\mathrm{Pb}$ collisions at $\sqrt{s_{\mathrm{NN}}}=5.02 \mathrm{TeV}$ as a function of (left) $p_{\mathrm{T}}$ for five centrality intervals, (right) $N_{\text {part }}$ for four transverse momentum ranges, [18]. 

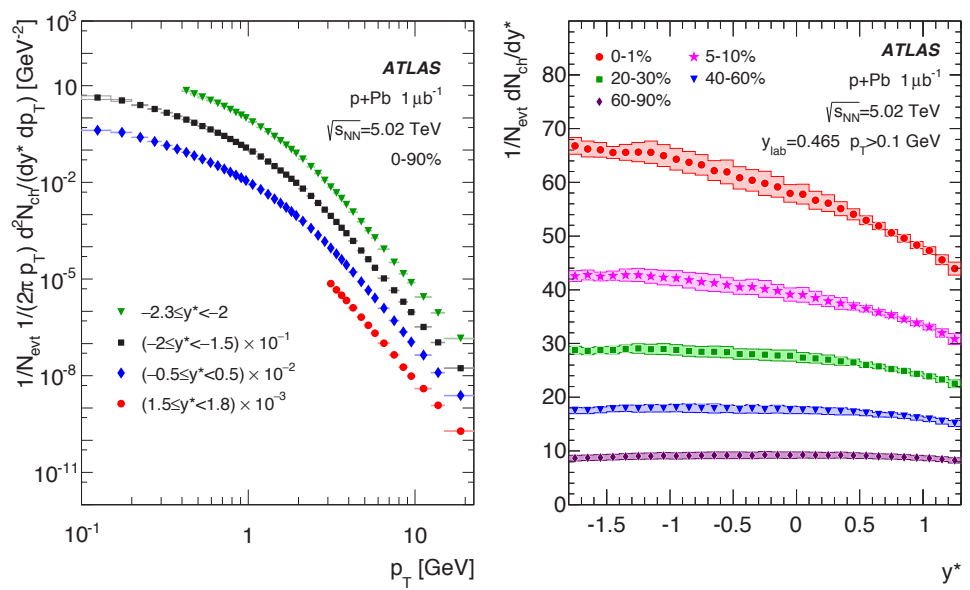

Figure 4. Invariant differential spectra of charged particles which are produced in $p+\mathrm{Pb}$ collisions at $\sqrt{s_{\mathrm{NN}}}=5.02 \mathrm{TeV}$ as a function of (left) transverse momentum $p_{\mathrm{T}}$, shown in four $y^{\star}$ ranges, for the $0-90 \%$ centrality interval (the individual spectra are scaled by constant factors indicated in the legend for better visibility), (right) rapidity $y^{\star}$, shown in five centrality intervals for $p_{\mathrm{T}}>0.1 \mathrm{GeV},[21]$.

is noticeably suppressed even in the $60-80 \%$ centrality interval but the suppression is strongest in the most central $0-5 \%$ collisions. Above this $p_{\mathrm{T}}, R_{\mathrm{AA}}$ generally increases with increasing $p_{\mathrm{T}}$ up to $\sim 60 \mathrm{GeV}$ after which the slope changes. This behavior is consistent with the analogous measurements made by the ATLAS experiment at $\sqrt{s_{\mathrm{NN}}}=2.76 \mathrm{TeV}$ [20]. Figure 3(right) shows $R_{\mathrm{AA}}$ as a function of the mean number of participating nucleons, $\left\langle N_{\text {part }}\right\rangle$, in four momentum intervals: the local maximum of $R_{\mathrm{AA}} 1.7<p_{\mathrm{T}}<2 \mathrm{GeV}$, the local minimum of $R_{\mathrm{AA}} 6.7<p_{\mathrm{T}}<7.7 \mathrm{GeV}$; the high- $p_{\mathrm{T}}$ region $p_{\mathrm{T}}>60 \mathrm{GeV}$; and an intermediate region $26<p_{\mathrm{T}}<30 \mathrm{GeV}$. In all four momentum intervals $R_{\mathrm{AA}}$ decreases with $\left\langle N_{\text {part }}\right\rangle$, however the decrease is strongest for the minimum interval and is weakest in the high- $p_{\mathrm{T}}$ region.

Charged hadron spectra have been also measured by ATLAS in $p+\mathrm{Pb}$ collisions at $\sqrt{s_{\mathrm{NN}}}=$ $5.02 \mathrm{TeV}$ [21]. In this assymetric collision a $4 \mathrm{TeV}$ proton beam and a $1.57 \mathrm{TeV}$ per-nucleon ${ }^{208} \mathrm{~Pb}$ beam are collided resulting in a longitudinal rapidity boost by 0.465 units of the centre-of-mass system with respect to the ATLAS laboratory frame. This means that the ATLAS laboratory frame rapidity, $y$, and the $p+\mathrm{Pb}$ centre-of-mass system rapidity, $y^{\star}$, are related by $y^{\star}=y-0.465$.

The differential invariant yields of charged particles produced are presented as a function of charged-particle transverse momentum in Fig. 4(left) for several intervals of $y^{\star}$. In Fig. 4(right) the invariant charged-particle yield as a function of $y^{\star}$ is shown for $p_{\mathrm{T}}>0.1 \mathrm{GeV}$ in several centrality intervals. In collisions that are more central, the charged-particle yields become progressively more asymmetric, with more particles produced in the Pb-going direction than in the proton-going direction. The measured nuclear modification factors (not shown) are observed to increase with transverse
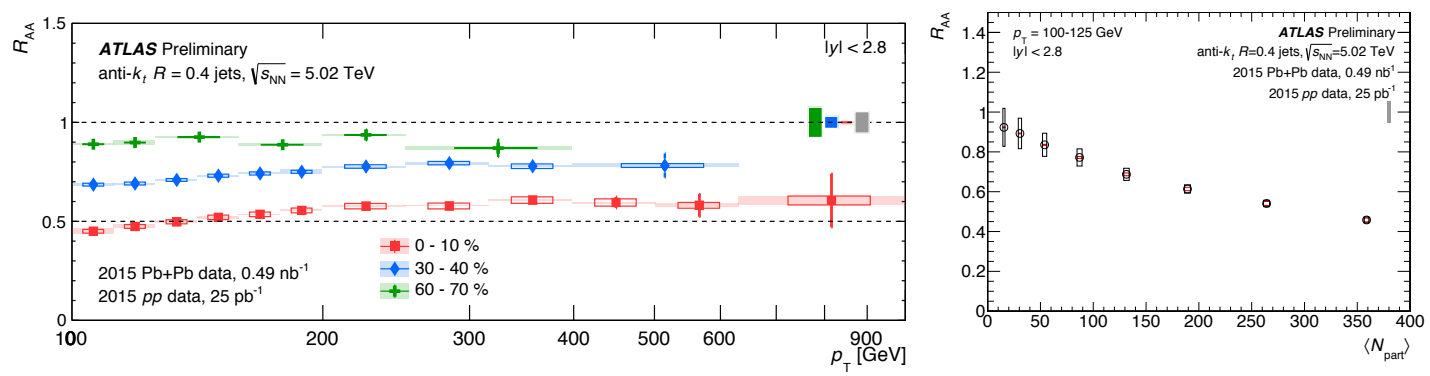

Figure 5. Nuclear modification factor $R_{\mathrm{AA}}$ (left) as a function of jet $p_{\mathrm{T}}$ for jets with $|y|<2.8$ for three centrality selections. (right) for jets with $p_{\mathrm{T}}=100-125 \mathrm{GeV}$ and $|y|<2.8$ evaluated as a function of $\left\langle N_{\text {part }}\right\rangle$, [22]. 

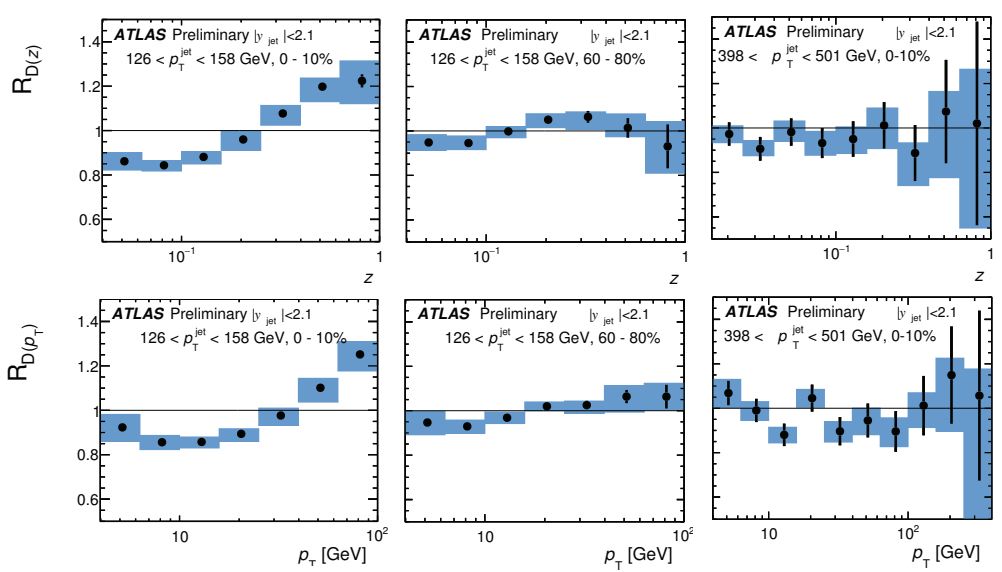

Figure 6. Ratios of fragmentation functions in $\mathrm{Pb}+\mathrm{Pb}$ and $p p$ collisions at $\sqrt{s_{\mathrm{NN}}}=5.02$ $\mathrm{TeV}$ as a function of (top) variable $z$, and (bottom) tracks transverse momentum $p_{\mathrm{T}}$ for $\left|y_{\text {jet }}\right|<2.1$ in two centrality selections (central $0-10 \%$ and peripheral $60-80 \%)$ and three $p_{\mathrm{T}}^{\mathrm{jet}}$ intervals as indicated in the plots, [25].

momentum from $0.1 \mathrm{GeV}$ to a peak value at $p_{\mathrm{T}} \approx 3 \mathrm{GeV}$, at which point they decrease slowly up to $p_{\mathrm{T}} \approx 8 \mathrm{GeV}$. Above this value they are constant within the experimental uncertainties.

Nuclear modification factors for jet production in $\mathrm{Pb}+\mathrm{Pb}$ collisions at $\sqrt{s_{\mathrm{NN}}}=5.02 \mathrm{TeV}$ have been studied by the ATLAS experiment in Ref. [22]. These new results extend the previous measurements to significantly higher transverse momenta and larger rapidities of jets. In this analysis and also in the following ones discussed below, to reconstruct jets ATLAS uses the anti- $k_{t}$ algorithm [23] with the radius parameter $R=0.4$. In Fig. 5(left) the jet $R_{\mathrm{AA}}$ is shown as a function of $p_{\mathrm{T}}$ in three centrality selections. The $R_{\mathrm{AA}}$ is evaluated for jets with $p_{\mathrm{T}}$ in the interval of $100-1000 \mathrm{GeV}$ and $|y|<2.8$. A clear suppression of the jet production in central $\mathrm{Pb}+\mathrm{Pb}$ collisions with respect to $p p$ collisions can be seen. In $0-10 \%$ central collisions the $R_{\mathrm{AA}}$ is approximately 0.45 near $p_{\mathrm{T}}=100 \mathrm{GeV}$. The $R_{\mathrm{AA}}$ is observed to grow slowly with increasing jet momentum reaching a value of approximately 0.6 for jets with $p_{\mathrm{T}}$ around $800 \mathrm{GeV}$. The $R_{\mathrm{AA}}$ evaluated for jets with $|y|<2.1$ is in agreement with a previous ATLAS measurement performed at $\sqrt{s_{\mathrm{NN}}}=2.76 \mathrm{TeV}$ [24]. The $\left\langle N_{\text {part }}\right\rangle$ dependence of the $R_{\mathrm{AA}}$ is shown in Fig. 5(right) for jets with $|y|<2.8$ and $100<p_{\mathrm{T}}<125 \mathrm{GeV}$. A smooth evolution of the $R_{\mathrm{AA}}$ is seen with the largest values in the most peripheral collision and the smallest values in the most central collisions.

Measurements of jet fragmentation functions into charged particles in $\mathrm{Pb}+\mathrm{Pb}$ and $p+\mathrm{Pb}$ collisions, both at $\sqrt{s_{\mathrm{NN}}}=5.02 \mathrm{TeV}$, have been measred by the ATLAS experiment in Refs. [25] and [26], respectively. Results are presented as a function of both charged particle transverse momentum with respect to the beam direction, $p_{\mathrm{T}}^{\text {trk }}$, and longitudinal momentum fraction relative to the jet axis $z \equiv$ $p_{\mathrm{T}}^{\mathrm{trk}} \cos \Delta R / p_{\mathrm{T}}^{\mathrm{jet}}$ and are written as: $D(z)=\left(1 / N_{\mathrm{jet}}\right)\left(\mathrm{d} N_{\mathrm{ch}} / \mathrm{d} z\right)$ and $D\left(p_{\mathrm{T}}\right)=\left(1 / N_{\mathrm{jet}}\right)\left(\mathrm{d} N_{\mathrm{ch}} / \mathrm{d} p_{\mathrm{T}}^{\mathrm{trk}}\right)$, where $\Delta R=\sqrt{\Delta \eta^{2}+\Delta \phi^{2}}$ and $N_{\text {ch }}$ and $N_{\text {jet }}$ are the numbers of charged particles and jets under consideration, respectively. To quantify the difference between fragmentation functions in $\mathrm{HI}$ collisions and $p p$ collisions the ratios of $D\left(p_{\mathrm{T}}\right)$ and $D(z)$ distributions measured in HI collisions to those measured in $p p$ collisions, $R_{D\left(p_{\mathrm{T}}\right)}$ and $R_{D(z)}$, are evaluated. The ratios for $\mathrm{Pb}+\mathrm{Pb}$ collisions at $\sqrt{s_{\mathrm{NN}}}=5.02 \mathrm{TeV}$ are shown in Fig. 6 for two centrality selections (central $0-10 \%$ and peripheral $60-80 \%$ ) and in three ranges of jets transverse momenta. Suppression of $R_{D(z)}\left(R_{D\left(p_{\mathrm{T}}\right)}\right)$ at intermediate $z\left(p_{\mathrm{T}}\right)$ and enhancement at high $z\left(p_{\mathrm{T}}\right)$ are observed in the data for central collisions. Both these features are less pronounced in peripheral collisions or for the jets with highest $p_{\mathrm{T}}^{\text {jet }}>398 \mathrm{GeV}$.

The ratios for $p+\mathrm{Pb}$ collisions at $\sqrt{s_{\mathrm{NN}}}=5.02 \mathrm{TeV}$ are shown in Fig. 7 in three intervals of jets transverse momenta. There is no modification of the jet fragmentation functions with respect to $p p$ onlliciono naithar os of funntion of tha rorishla - non $n$ 

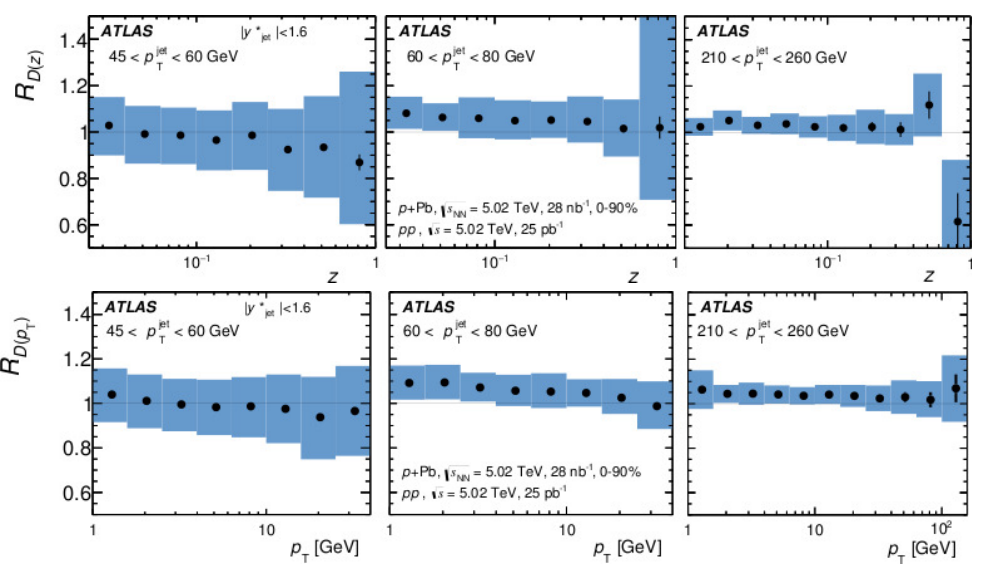

Figure 7. Ratios of fragmentation functions in $p+\mathrm{Pb}$ and $p p$ collisions at $\sqrt{s_{\mathrm{NN}}}=5.02 \mathrm{TeV}$ as a function of (top) variable $z$, and (bottom) track transverse momentum $p_{\mathrm{T}}$ for $\left|y_{\text {jet }}^{\star}\right|<1.6$ for three $p_{\mathrm{T}}^{\text {jet }}$ intervals as indicated in the plots, [26].

\section{Bulk collectivity}

One of the signatures of the collective behaviour of the hot dense medium produced in HI collisions is the azimuthal anisotropy of produced particles. This anisotropy results from spatial asymmetry in the initial interaction region which activates strong pressure gradients along the shorter axis of the overlap region, leading to increased production of particles within the reaction plane, defined by the impact parameter vector (the vector separation of the barycentres of the two nuclei) and the beam axis. The azimuthal anisotropy is commonly characterized by Fourier harmonics $\mathrm{v}_{n}$, referred to as single-particle harmonic flow coefficients: $\mathrm{v}_{n}=\cos \left[n\left(\phi-\Phi_{R}\right)\right]$, where $\phi$ is the azimuthal angle of a particle and $\Phi_{R}$ is the azimuthal angle of the reaction plane. This anisotropic, collective enhancement of particle production is a global long-range phenomenon extending over a wide pseudorapidity range.

The anisotropy of charged-particle azimuthal angle distributions in $\mathrm{HI}$ collisions has been a subject of extensive experimental studies at the LHC. Recently a measurement of multi-particle azimuthal correlations in $p p, p+\mathrm{Pb}$ at $\sqrt{s_{\mathrm{NN}}}=5.02 \mathrm{TeV}$ and low-multiplicity $\mathrm{Pb}+\mathrm{Pb}$ collisions at $\sqrt{s_{\mathrm{NN}}}=2.76 \mathrm{TeV}$ has been performed by ATLAS [27]. The Fourier harmonics are studied as a function of the chargedparticle multiplicity. In order to avoid influence of event-by-event multiplicity fluctuations, the comparison between different systems is made based on the linear correlation between the number of charged particles with $|\eta|<2.5$ and $0.3<p_{\mathrm{T}}<3 \mathrm{GeV}$ (referred to as $M_{\text {ref }}$ selection) and the average number of charged particles with $p_{\mathrm{T}}>0.4 \mathrm{GeV},\left\langle N_{\mathrm{ch}}\left(p_{\mathrm{T}}>0.4\right)\right\rangle$. The comparison between different systems of $\mathrm{v}_{i}\{2,|\Delta \eta|>2\}, i=1,2,3$ obtained from two-particle cumulants built from charged tracks separated in rapidity by $|\Delta \eta|>2$ is shown in Fig. 8. It is seen that for the same multiplicity,
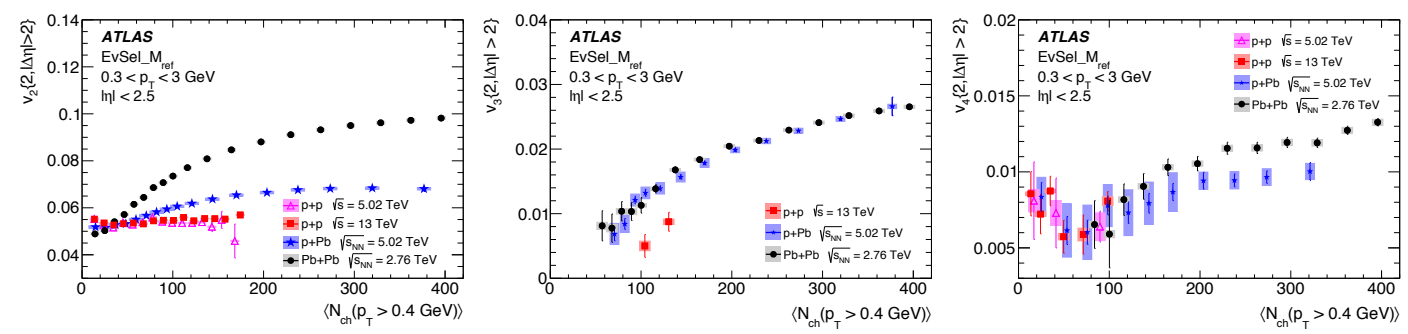

Figure 8. Comparison of (left) $\mathrm{v}_{2}\{2,|\Delta \eta|>2\}$, (middle) $\mathrm{v}_{3}\{2,|\Delta \eta|>2\}$, and (right) $\mathrm{v}_{4}\{2,|\Delta \eta|>2\}$, as a function of $\left\langle N_{\mathrm{ch}}\left(p_{\mathrm{T}}>0.4 \mathrm{GeV}\right)\right\rangle$ for $p p$ collisions at $\sqrt{s}=5.02$ and $13 \mathrm{TeV}, p+\mathrm{Pb}$ collisions at $\sqrt{s_{\mathrm{NN}}}=5.02 \mathrm{TeV}$ and low-multiplicity $\mathrm{Pb}+\mathrm{Pb}$ collisions at $\sqrt{s_{\mathrm{NN}}}=2.76 \mathrm{TeV}$ for $0.3<p_{\mathrm{T}}<3 \mathrm{GeV}$ range of reference particles, [27]. 
$\mathrm{v}_{2}\{2\}$ is higher for $\mathrm{Pb}+\mathrm{Pb}$ than for $p+\mathrm{Pb}$ (factor 2 at the highest multiplicities). The smallest values of $\mathrm{v}_{2}\{2\}$ are observed for $p p$ collisions with no dependence on collision energy nor on multiplicity. $\mathrm{v}_{3}\{2\}$ for $\mathrm{Pb}+\mathrm{Pb}$ and $p+\mathrm{Pb}$ systems are similar and much higher than for $13 \mathrm{TeV} p p$ data. For all systems $v_{3}\{2\}$ increases with multiplicity. Weaker increase with multiplicity is seen for $v_{4}\{2\}$, but at high multiplicities the values for $\mathrm{Pb}+\mathrm{Pb}$ are systematicaly larger than for $p+\mathrm{Pb}$ collisions.

\section{Ultra-peripheral collisions}

In the case of nuclei colliding at large impact parameters, the strong interaction is not active. However, due to their strong electromagnetic fields, the nuclei can coherently produce high energy nearly real photons. That means that in these so-called ultra-peripheral collisions (UPC) one can study twophoton processes, because relatively small couplings are compensated with very high photon-photon luminosity which scales with the nuclear electric charge as $Z^{4}$. The maximum energy for coherent photons emitted from relativistic nucleus is $\gamma \hbar c / R$, where $\gamma$ is the Lorentz factor of one nucleus and $R$ is the nuclear radius. This is about $75 \mathrm{GeV}$ for the $\mathrm{Pb}+\mathrm{Pb}$ collisions at $\sqrt{s_{\mathrm{NN}}}=5.02 \mathrm{TeV}$.

The first direct measurement of the light-by-light scattering process, $\gamma \gamma \rightarrow \gamma \gamma$, has been done by ATLAS in $\mathrm{Pb}+\mathrm{Pb}$ collisions at $\sqrt{s_{\mathrm{NN}}}=5.02 \mathrm{TeV}$ [28]. This process is forbidden in classical electrodynamics, and in QED, it proceeds at thee lowest order in the fine structure constant $\left(\alpha_{\mathrm{em}}\right)$ via virtual one-loop box diagrams involving fermions or charged bosons, which is an $O\left(\alpha_{\mathrm{em}}^{4} \approx 3 \times\right.$ $10^{-9}$ ) process, making it challenging to test experimentally. The final-state signature is the exclusive production of two photons, $\mathrm{Pb}+\mathrm{Pb}(\gamma \gamma) \rightarrow \mathrm{Pb}^{\star}+\mathrm{Pb}^{\star}+\gamma \gamma$ (see Fig. 9(left)), where a possible electromagnetic excitation of the outgoing ions is denoted by $(\star)$. Hence, the expected signature is two photons and no further activity in the central detector, since the $\mathrm{Pb}$ ions escape into the LHC beam pipe. The kinematic distributions of $\gamma \gamma \rightarrow \gamma \gamma$ event candidates are shown in Fig. 9 together with signal and background predictions. The statistical significance against the background-only hypothesis is estimated to be 4.4 standard deviations. The measured fiducial cross section in the phase space defined by the photon transverse energy $E_{\mathrm{T}}>3 \mathrm{GeV}$, photon pseudorapidity $|\eta|<2.4$, diphoton invariant mass $m_{\gamma \gamma}>6 \mathrm{GeV}$, diphoton transverse momentum $p_{\mathrm{T}}^{\gamma \gamma}<2 \mathrm{GeV}$ and diphoton acoplanarity below 0.01 , is $\sigma_{\text {fid }}=70 \pm 24($ stat $) \pm 17$ (syst) nb, which is in agreement with the predicted by Standard Model values of $45 \pm 9 \mathrm{nb}$ [29] and $49 \pm 10 \mathrm{nb}$ [30] within uncertainties.

Photo-nuclear dijet production has been studied by ATLAS in ultra-peripheral $\mathrm{Pb}+\mathrm{Pb}$ collisions at $\sqrt{s_{\mathrm{NN}}}=5.02 \mathrm{TeV}$ [31]. The dijet production can proceed via 'direct' photo-production or 'resolved' photon processes. In direct photo-production the photon acts like a point-like object interacting with
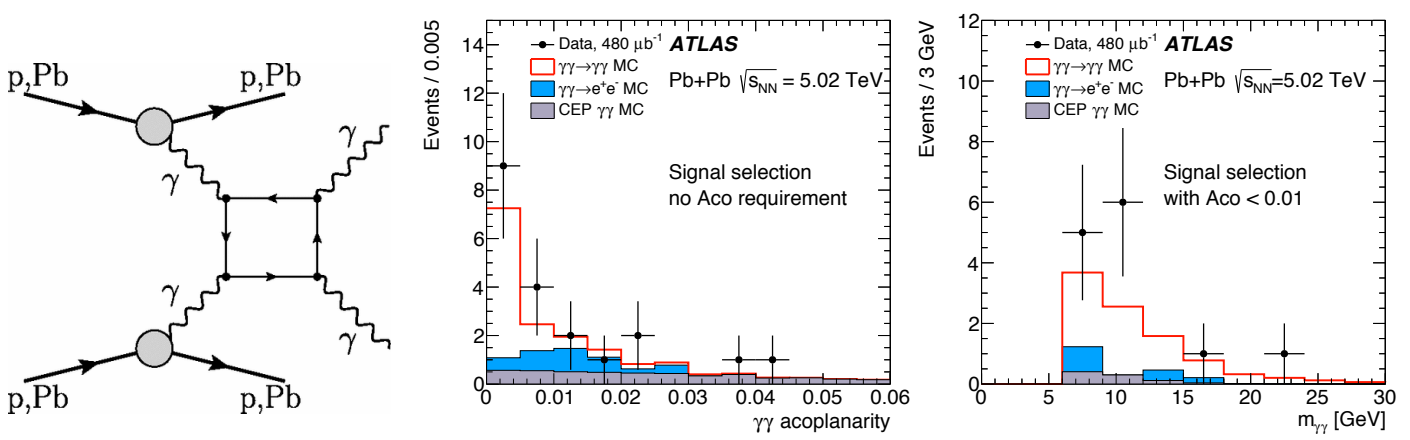

Figure 9. (left) Schematic view of the light-by-light scattering process. (middle) Diphoton acoplanarity before applying Aco $<0.01$ requirement. (right) Diphoton invariant mass after applying Aco $<0.01$ requirement, [28]. 
the partons in the other nucleus. However, resolved processes may also occur in which virtual excitations of the photon may also be probed by the hard interaction in which case the photon serves as a source of partons. In this contribution only a fraction of the photon's four-momentum contributes to the hard scattering. As neither type of process involves the exchange of colour with the photonemitting nucleus, no significant particle production is expected in the rapidity region between the dijet system and that nucleus. Thus a rapidity gap is expected, and a requirement of a minimum rapidity gap may be used to separate photo-nuclear events from hadronic $\mathrm{Pb}+\mathrm{Pb}$ collisions. The gap is expected to be smaller in resolved photon events due to the additional particle production associated with the photon remnant. The photon-emitting nucleus is also expected to produce few or no neutrons because the photon flux is largest for momenta in the range where they couple to the entire nucleus. The emission of such photons is not expected to excite the nucleus. Thus a combination of a rapidity gap and zero neutrons in the same direction provide straightforward criteria to identify these events experimentally. However, additional soft photon exchanges during the ultra-peripheral collision can cause the photon-emitting nucleus to be excited into low-lying states where it may emit a small number of evaporation neutrons and a measurement must either correct for this effect or be compared to a theoretical model that includes it.

The differential cross sections for dijet production have been measured in terms of the following variables: $H_{\mathrm{T}}=\Sigma_{\text {jets }} p_{\mathrm{T}}^{\text {jet }}, z_{\gamma}=\left(m_{\text {jets }} / \sqrt{s}\right) \exp \left(y_{\text {jets }}\right)$ and $x_{\mathrm{A}}=\left(m_{\text {jets }} / \sqrt{s}\right) \exp \left(-y_{\text {jets }}\right)$, where $m_{\text {jets }}$ and $y_{\text {jets }}$ are invariant mass and rapidity of the dijet system. In the limit of $2 \rightarrow 2$ scattering kinematics, $x_{\mathrm{A}}$ corresponds to the ratio of the energy of the struck parton in the nucleus to the (per nucleon) beam energy, while $z_{\gamma}=x_{\gamma} y$, where $\mathrm{y}$ is the energy fraction carried by the photon. For direct processes, $x_{\gamma}$ is unity, while for resolved events, it is the fraction of the photon's energy carried by the resolved parton entering the hard scattering.

The measured double-differential cross sections for dijet production are shown in Fig. 10(left) as a function of $H_{\mathrm{T}}$ and $x_{\mathrm{A}}$, and in Fig. 10(right) as a function of $z_{\gamma}$ and $H_{\mathrm{T}}$. The shapes of the distributions are well described by the Pythia+Starlight MC [32].

\section{Summary}

Recent results from the ATLAS experiment related to the study of Quark-Gluon Plasma via both hard and soft probes have been briefly presented. For more details and in depth discussion of the measurements the reader is referred to the original publications. All ATLAS heavy-ion results are

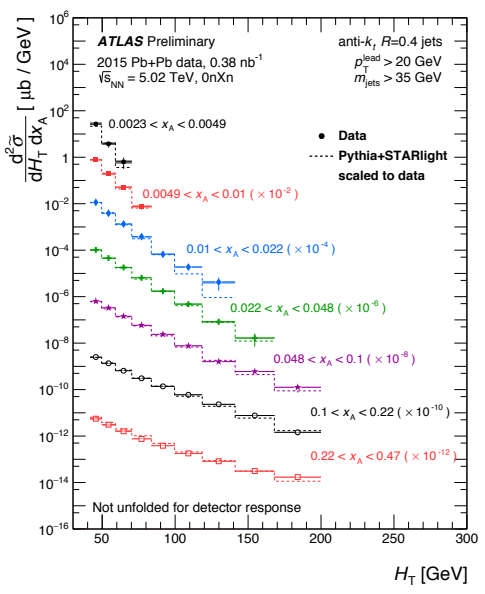

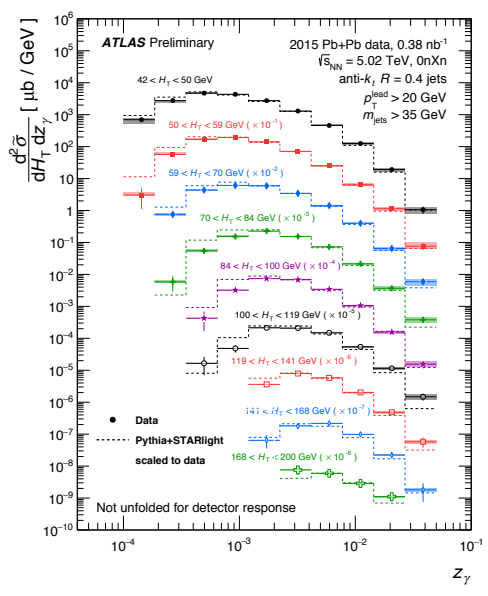

Figure 10. (left) Double differential cross section $d^{2} \tilde{\sigma} / d H_{\mathrm{T}} d x_{\mathrm{A}}$ as a function of $H_{\mathrm{T}}$ for different bins of $x_{\mathrm{A}}$. (right) Double differential cross section $d^{2} \tilde{\sigma} / d H_{\mathrm{T}} d z_{\gamma}$ as a function of $z_{\gamma}$ for different bins of $H_{\mathrm{T}}$. The cross sections are scaled by powers of 10 to improve visibility. The dashed lines represent the cross section from Pythia+Starlight scaled to have the same integral as the data within the fiducial region of the measurement, [31]. 
available from https://twiki.cern.ch/twiki/bin/view/AtlasPublic/HeavyIonsPublicResults This work was partly supported by the National Science Centre of Poland under grant number UMO2016/23/B/ST2/01409 and by PL-GRID infrastructure.

\section{References}

[1] STAR Collaboration, Nucl. Phys. A757, 102 (2005), [nucl-ex/0501009].

[2] BRAHMS Collaboration, Nucl. Phys. A757, 1 (2005), [nucl-ex/0410020].

[3] PHOBOS Collaboration, Nucl. Phys. A757, 28 (2005), [nucl-ex/0410022].

[4] PHENIX Collaboration, Nucl. Phys. A757, 184 (2005), [nucl-ex/0410003].

[5] N. Armesto and E. Scomparin, Eur. Phys. J. Plus 131, 52 (2016), [nucl-ex/1511.02151].

[6] ATLAS Collaboration, JINST 3 (2008) S08003, https://cds.cern.ch/record/1129811.

[7] ATLAS Collaboration, Phys. Lett. B707 (2012) 330-348, [nucl-ex/1108.6018].

[8] ATLAS Collaboration, Eur. Phys. J. C76 (2016) 199, [hep-ex/1508.00848].

[9] V. Guzey, M. Strikman, Phys. Lett. B633 (2006), [hep-ph/0505088].

[10] K. Eskola et al., J. High Energy Phys. 04, 065 (2009), [hep-ph/0902.4154].

[11] ATLAS Collaboration, Eur. Phys. J. C75 (2015) 23, [hep-ex/14084674].

[12] ATLAS Collaboration, Phys. Rev. Lett. 110, 022301 (2013), [hep-ex/1210.6486].

[13] ATLAS Collaboration, ATLAS-CONF-2015-056, http://cds.cern.ch/record/2055677.

[14] ATLAS Collaboration, Phys. Rev. C92, 044915 (2015), [hep-ex/1507.06232].

[15] ATLAS Collaboration, ATLAS-CONF-2017-010, http://cds.cern.ch/record/2244821.

[16] T. Matsui and H. Satz, Phys. Lett. B178 (1986) 416.

[17] ATLAS Collaboration, ATLAS-CONF-2016-109, https://cds.cern.ch/record/2220771.

[18] ATLAS Collaboration, ATLAS-CONF-2017-012, https://cds.cern.ch/record/2244824.

[19] J.W. Cronin et al., Phys. Rev. D11 (1975) 3105.

[20] ATLAS Collaboration, J. High Energy Phys. 09, 50 (2015), [hep-ex/1504.04337].

[21] ATLAS Collaboration, Phys. Lett. B763 (2016) 313, [hep-ex/1605.06436].

[22] ATLAS Collaboration, ATLAS-CONF-2017-009, https://cds.cern.ch/record/2244820.

[23] M. Cacciari, G.P. Salam, G. Soyez, J. High Energy Phys. 04, 063 (2008), [hep-ph/0802.1189].

[24] ATLAS Collaboration, Phys. Rev. Lett. 114, 072302 (2015), [hep-ex/1411.2357].

[25] ATLAS Collaboration, ATLAS-CONF-2017-005, https://cds.cern.ch/record/2244802.

[26] ATLAS Collaboration, submitted to Phys. Lett. B, [hep-ex/1706.02859].

[27] ATLAS Collaboration, Eur. Phys. J. C77 (2017) 428, [hep-ex/1705.04176].

[28] ATLAS Collaboration, Nature Physics 13 (2017) 852, [hep-ex/170201625].

[29] D. d'Enterria and G. G. Silveira, Phys. Rev. Lett. 111, 080405 (2013), [Erratum: Phys. Rev. Lett. 116, 129901 (2016)], [hep-ph/1305.7142].

[30] M. Klusek-Gawenda, P. Lebiedowicz, and A. Szczurek, Phys. Rev. C93 (2016) 044907, [hepth/1601.07001].

[31] ATLAS Collaboration, ATLAS-CONF-2017-11, https://cds.cern.ch/record/2244822.

[32] S. Klein and J. Nystrand, The STARLIGHT website, http://starlight.hepforge.org. 